\title{
How do policy advisors and practitioners prioritise the protection of children from secondhand smoke exposure in a country with advanced tobacco control policy?
}

\author{
Deborah Doreen Ritchie, ${ }_{1}^{1}$ Amanda Amos, ${ }^{2}$ April Shaw, ${ }^{3}$ Rachel O'Donnell, ${ }_{1}^{3}$ \\ Sean Semple, ${ }^{4}$ Steve Turner, ${ }^{5}$ Claudia Martin ${ }^{6}$
}

For numbered affiliations see end of article.

Correspondence to Dr Deborah Doreen Ritchie, School of Health in Social Science, University of Edinburgh, Teviot Place, Edinburgh EH98AG, UK ; Deborah.Ritchie@ed.ac.uk

Received 14 December 2012 Accepted 8 July 2013 Published Online First 16 August 2013

\begin{abstract}
Objectives The aim is to extend understanding of the policy and practice discourses that inform the development of national tobacco control policy to protect children from secondhand smoke exposure (SHSE) in the home, particularly in a country with successful implementation of smoke-free public places legislation. The Scottish experience will contribute to the tobacco control community, particularly those countries at a similar level of tobacco control, as normalising discourses about protecting children from SHSE are becoming more widespread.

Design Case study design using qualitative interviews and focus groups (FGs) with policy makers, health and childcare practitioners during which they were presented with the findings of the Reducing Families' Exposure to Secondhand Smoke (REFRESH) intervention and discussed the implications for their policy and practice priorities.

Setting Scotland, UK

Participants Qualitative interviews and FGs were conducted with 30 policy makers and practitioners who were purposively recruited.

Results Participants accepted the harm of SHSE to children; however, action is limited by political expedience due to- - the perception of a shift of the public health priority from smoking to alcohol, current financial constraints, more immediate child protection concerns and continuing unresolved ethical arguments.

Conclusions In a country, such as Scotland, with advanced tobacco control strategies, there continue to be challenges to policy and practice development in the more contentious arena of the home. Children's SHSE in their homes is unequivocally accepted as an important health priority, but it is not currently perceived to be a top public health priority in Scotland.
\end{abstract}

\section{INTRODUCTION}

Considerable progress has been made in several countries in reducing children's exposure to secondhand smoke (SHS) following the implementation of smoke-free public places legislation. ${ }^{1-3}$ This article explores the continuing barriers to protecting children from SHS exposure (SHSE) in the home and draws upon Scotland as a case study within the UK, a country which has successful, comprehensive smoke-free legislation, increasing social denormalisation of smoking and is top of the European Scale for Tobacco Control. ${ }^{4-7}$ The Scottish experience will contribute to the tobacco control community in countries with similar levels of tobacco control where normalising discourses about protecting children from SHSE are becoming more widespread. ${ }^{8}$

The development of smoke-free public places-a cornerstone of recent Scottish tobacco control policy -was informed by the growing international consensus about the serious harm caused to non-smoking adults and children from involuntary exposure to SHS. ${ }^{9-11}$ The U.S. Surgeon General's Reports (1986, 2006) reached important conclusions about how SHSE is particularly harmful and hazardous to children's health. ${ }^{9}{ }^{10}$ Scottish children are now protected from SHSE in enclosed public places and thus most children's SHSE occurs in the home and car. Children, particularly younger children, can find it difficult to avoid this SHSE, reflecting constrained living conditions and lack of individual autonomy, and are therefore more likely to be more heavily exposed than other age groups. ${ }^{11} 12$

Following the implementation of smoke-free legislation in Scotland, interest in the spatial boundaries of smoke-free environments began to shift from the public domain to the private domain of the home. ${ }^{11}$ Changing attitudes towards smoking in the home were shaped, in part, by the recent social denormalisation strategies of the smoke-free legislation $^{211} 13$ and also by emerging lay understandings of the harms caused by SHSE, including increasing acceptance that young children should be protected from SHS. ${ }^{11} 14$ It has been suggested that populations become more amenable to smoking restrictions in the home as smoke-free environments become more widespread. ${ }^{111}$

The smoke-free legislation in Scotland produced significant health benefits for smokers, non-smokers, children and workers previously exposed to SHS. ${ }^{5} 1516$ Smoking in the home did not increase after implementation of the legislation and children's exposure to SHS reduced. ${ }^{2}{ }^{17}$ Overall, SHSE fell by 39\% between January 2006 and January $2007^{2}$ with the greatest reductions in children in homes with lower SHSE. ${ }^{2}$ However, children's SHSE is still significant, particularly among disadvantaged children and/or where the mother or both parents smoke. ${ }^{17} \mathrm{In}$ Scotland, $65 \%$ of children from lower socioeconomic groups had one or both parents who smoked compared with $35 \%$ of children from more affluent groups. ${ }^{17}$ Therefore, attention has been focused on how to reduce their exposure in the home, which is the main source of SHSE. ${ }^{12}$ Despite the normalising discourse about the need to protect children from SHSE, recent qualitative research has highlighted that 
several barriers to creating smoke-free homes, related to gender and social and environmental contexts, still exist particularly in disadvantaged homes. ${ }^{11} 141819$

The continuing public health need for a strategic approach to supporting families to reduce SHS in the home seems clear. However, research in countries with smoke-free legislation has identified several barriers to this. New Zealand researchers explored policy makers' views of smoke-free private places and identified that key drivers for not introducing state enforcement were the lack of political acceptability and the feasibility of intervening in the private space. ${ }^{8}$ Research that explored the views of tobacco control professionals in Scotland in the year following the smoke-free legislation found that they prioritised the need for smoke-free home interventions in more disadvantaged communities where smoking at home was most prevalent. ${ }^{20}$ However, they also identified several ethical challenges that needed to be addressed, particularly the balance between the welfare of the child to be protected from SHSE against parents not being stigmatised by their smoking. In addition, they considered the ethics of interventions for parents who had significant social and environmental barriers to overcome. Other arguments concerned the private space of the home and parents' right to smoke in their homes. ${ }^{20}$

Many countries are at the same stage as Scotland with strong policies limiting SHSE in public places. This article provides an analysis of the current debates around progressing policy and practice that apply to private spaces, that is, the home. The aim is to extend our understanding of stakeholders' views of SHSE (policy advisors, health professionals and childcare practitioners) and draw upon different stakeholders discourses' to explore how they rationalise the prioritisation of the SHSE risk to children and the desirability and feasibility of further action on this issue. This article considers the perceived relationship of SHSE to other child protection priorities, and how further action to reduce children's SHSE can be constrained by limited financial resources and shifting public health priorities. This article is viewed through the lens of the Reducing Families' Exposure to Secondhand Smoke (REFRESH) project, which was a feasibility study that evaluated a smoke-free home intervention delivered in the home to mothers who smoked and had a child under 6 years old. ${ }^{21-23}$ This article draws on data from phase III of the study, which explored how policy makers and public health practitioners responded to the positive evidence from REFRESH on the feasibility of a smoke-free home intervention, the risks of SHSE to children in general and the implications of the findings for their policy and practice. This also generated discussions on the current policy milieu and the potential to further the agenda more radically by policy and practice.

\section{METHODS}

\section{Study design}

REFRESH was a novel smoke-free home intervention using an empowerment approach, carried out in Grampian (Scotland), which involved 54 smoking mothers with at least one child under 6 years old. It aimed to reduce children's SHSE in their homes by providing personalised feedback on home air quality in the context of a motivational interview. The study had three phases: phase I-intervention delivery and evaluation, ${ }^{21-23}$ phase II-qualitative evaluation with intervention participants $^{21} 22$ and phase III-interviews with policy makers and practitioners. This article considers data from phase III.

\section{Participants}

The sample was selected to reflect the diversity of stakeholders who shape policy and practice at national and local levels in Scotland.
Table 1 Phase III: sample

\begin{tabular}{lcl}
\hline Sector of participants & $\begin{array}{l}\text { Number of } \\
\text { participants }\end{array}$ & Interview or FG \\
\hline $\begin{array}{l}\text { Scottish government policy } \\
\text { advisors }\end{array}$ & 3 & interviews \\
NHS & & \\
$\quad$ Smoking cessation workers & 4 & Interviews \\
$\quad$ Nurse managers/primary care & 20 & $\begin{array}{l}\text { focus groups } \\
\text { (n=4) }\end{array}$ \\
Local government & 2 & interviews \\
National body & 1 & interview \\
Total & 30 & \\
\hline
\end{tabular}

FG, focus group; NHS, National Health Service.

Thirty participants were purposively recruited. Individual interviews and focus groups (FGs) were conducted with a range of stakeholders (table 1). Scottish government participants were drawn from public health, early years and tobacco control policy teams. One National Health Service (NHS) FG included senior nurse managers with operational responsibility for community nursing in a health board area. The other three NHS Primary Care FGs were conducted in three localities, two in deprived urban areas and one in a small rural town with a socioeconomically mixed population. Participants included general practitioners, health visitors, practice nurses, practice managers, healthcare assistants and a medical student. Smoking cessation workers from two health board areas were interviewed. Local government participants were group workers in Children and Family Centres. The director of a national body that focuses on children's well-being was also interviewed.

\section{Data collection methods}

The study used qualitative methods using a topic guide to explore participants' views. The data were collected between December 2011 and May 2012. All the participants had previously received information about REFRESH. The interviewer first described how the REFRESH intervention was conducted and the main findings (box 1). This was followed by a semistructured interview or FG covering:

- Perceptions of the importance of children's exposure to SHS in their organisation and their professional practice.

- Views about the REFRESH intervention and its potential utility.

\section{Box 1 REFRESH key findings presented to policy and} practice participants (phase III)

REFRESH key findings

1. Providing mothers who smoke with personalised data about the air quality in their homes, together with a motivational interview, is feasible and has an effect on improving air quality measures at 1 month.

2. Knowledge about secondhand smoke (SHS) exposure among these mothers was limited. Increasing mothers' awareness of the risks can be shocking (as described by the mothers in the REFRESH study), but providing personalised data with immediate support to overcome perceived barriers is empowering in helping them reduce SHS in their homes.

3. The intervention was understandable and acceptable.

REFRESH, Reducing Families' Exposure to Secondhand Smoke. 
- Perceptions of the barriers to SHS work in their organisation/practice and in other sectors.

- Perceptions of opportunities for SHS work in their own organisation/practice and in other sectors.

- Views about who should be responsible for this area of activity.

The choice of using FGs or interviews was partly pragmatic due to the difficulties of securing time with busy professionals. However, the interviews allowed for probing in more depth and were therefore appropriate for high-level policy advisors. The FGs reflected team approaches in primary care.

\section{Data analysis}

The FGs and interviews, apart from one, were digitally recorded and transcribed. Notes were taken during the interview that could not be recorded because of background noise. Throughout this article, where quotes are used, the sector, participant's role and whether it was a FG or interview (I) are indicated.

The aim of the analytical approach was to comprehensively map the range and diversity of participants' narratives. The transcribed material was subject to systematic thematic analysis using the framework approach. ${ }^{24}$ Based on a thorough reading of the transcripts by the research team, a thematic coding frame was developed from the emergent themes: perceptions of SHS and its importance, perceptions of the REFRESH intervention and findings, barriers to SHS work professional and organisational factors, familial and sociocultural factors and opportunities for SHS work and drivers of change. Each transcript was coded and summarised within that coding frame. The key points within each coding category for each respondent were identified and a chart was created which drew together the key points for each respondent by thematic code. The next analytical stage entailed developing higher order themes within and across individual charts: the acceptance of the harm of SHSE to children, the nature of acceptable action, prioritisation processes, child protection and ethical issues.

\section{Findings}

Acceptance of harm of SHSE to children

Policy advisors and practitioners were unequivocal in their acceptance of the evidence that SHSE was a serious risk to children's health. Practitioners clearly recognised the harm to health, but were aware that they did not always look for the harm. Policy makers accepted that it should be a key part of a public health strategy.

[SHS Is] very important, because we know that it affects children's health, it also makes a difference to what they do longer term, and because we work in a deprived population a lot of our patients already are at a higher risk of all sorts of illnesses, we've got a higher risk of prevalence of smoking as well. (NHS, Primary Care, FG)

I suspect we probably don't look for it enough but it is clearly important. Children are exposed at all stages. From in-utero-it's normal to be exposed. (NHS, Primary Care, FG)

It's very important and will always be a key part of the strategy. (Policy advisor, I)

\section{Acceptable action}

Both policy advisors and practitioners suggested policy and practice approaches to create smoke-free homes that would be acceptable and feasible. Practitioners focused on the opportunities to provide information and raise awareness that would help parents make empowered choices.
It's informed choices as well, and I firmly believe that, if someone's got a child that's had glue ear for example, and nobody has ever raised that their smoking in the home can have an impact on that, then, you can't really blame that person ...... if people have got the information, and they still choose to expose their children, then that's their informed choice, but I firmly believe that if they've no got the information, then you know, it's a shame really. (NHS, Smoking Cessation Practitioner, I)

This primary care participant also valued the acceptability of information, but located action within a broader public health approach.

This is information that could be used in various ways; it's also difficult to know what intervention is best (for individuals). The lesson from smoking cessation is that people go through a variety of ways to quit. The intervention needs to be at different levels-policy level, legislative level, ministerial level, GP level, a brief intervention in a 10 minute consultation. (NHS, Primary Care, FG)

Policy advisors supported the development of smoke-free home interventions, as long as it did not require any legislative input.

We know that the ban in 2006 did reduce children's exposure to second-hand smoke and there wasn't a corresponding transfer of exposure within the home. However, now the most likely places in which children are exposed are in the home and the car. The emphasis on SHS exposure within the home takes policy and action beyond the smoking ban into an area where it may not be possible or appropriate to legislate. Scottish Government is looking for interventions that may fill the gap that legislation may not be able to address, which it can promote. (Policy advisor, I)

\section{Political reluctance}

The potential for policy action appeared to be limited by political caution due to several reasons: the unacceptability of legislation to protect children from SHSE in the home or car, the perceived shift of public health priorities from smoking to alcohol and current financial constraints. It was argued that the private space of the home acted as a political barrier to further legislation to promote smoke-free spaces. Policy advisors in particular expressed a view that intervention in the home was seen as a step too far, despite the success of legislation on smoke-free public places.

There are issues around the extent to which there is freedom to smoke in private, you know, so that, so that there's a balance to be struck there, you know, how far can you go? How far can government go to impair the choice of what goes on inside people's cars or houses or whatever. (Policy advisor, I)

\section{Shifting public health priorities}

Although the claim was made that smoking was still a national priority, policy advisors in particular highlighted the increasing public health focus on alcohol as a key priority, and that the success of smoke-free public places provided a rationale for reprioritising resources.

It's not top of the agenda, I mean there are clearly all sorts of other issues live at the moment, there's limited parliamentary debating time and parliamentary process time and so on and at the moment probably the major public health intervention is around alcohol, there's a sense that okay, we've won significant progress in smoking, let's try and get alcohol under control ....so that is a higher priority, but the general sense of smoking being an issue that we need to keep on top of, is very strong with ministers, the question is what's the best thing to do about it? Because, you know, it's not a dictatorship. (Policy advisor, I) 
Financial constraints

The Scottish economic recession, with a focus on cuts in public spending and reductions in Scottish public sector services, was a consistent backdrop underpinning participant views. Thus, perhaps not surprisingly and despite the acceptance of harm to children, financial considerations were highlighted as constraints to developing new interventions to protect children from SHSE in the home. Participants across all sectors pointed out that there was no new money for smoke-free home interventions, that there were limited human and financial resources and this, in turn, had necessitated further prioritisation of activity. In the context of considering investing in a REFRESH intervention, one senior nurse manager (FG) commented that:

It might be possible but again that's still quite expensive, because I don't know how many smokers we have in [place], but we have just over 9000 births, so 9000 times $£ 200$, that's excessive, because not everybody will be a smoker, but it's a lot of money in a cash strapped environment, so I don't, I mean, it might, I think it's a very good idea, it clearly has some impact.

Int: Right, I mean, does the financial climate affect how much people can do, and how much health visitors?

Yeah, well it effects how much we can buy, because you know, we have a very limited supplies budget and a very limited staffing budget.

Other nurse managers concluded that smoking interventions were currently a low priority because of the impact of cuts on other services and the subsequent problems they faced dealing with basic childcare.

We were at a child protection event last week, and teachers are up against it, I mean, the very least they would be worrying about is the person smoking, actually, you know, they are worried about the child coming in and still in nappies in school, or haven't eaten, you know, so, you know, actually the priorities for them in terms of trying to get the parents to stop smoking is fairly low down, I would think, if the parents that are turning up are interested, even if they are smoking, that's a plus, you know, if they are getting their child to school, ......... and they've lost a lot of support because education has been cut, you know, a lot of the people who would have done some of those interventions aren't there anymore. (NHS, Senior nurse manager, I)

\section{More immediate child protection concerns and families with 'chaotic' lifestyles}

Competing professional priorities were identified in relation to policy and practice. Regarding policy, there was an unambiguous declaration — as has already been noted-that smoking was no longer perceived to be the key public health priority within government and that the focus was firmly on the alcohol issue. In terms of practice, the issue was framed somewhat differently around the demands of the professional role. A common narrative was that alcohol, drugs, domestic violence and child abuse were deemed to be more pressing.

I keep coming back to this, as I say the alcohol and the drugs, are probably seen by us as professionals to be more harmful, to young babies and to children, that really is the highest priority, you know, although as I say, I know smoking during pregnancy, and in the early years, even, is very detrimental to the children's brain development, but I think as I say, it's the alcohol and the drugs that do, in our eyes, a lot more damage, that wouldn't be right, I suppose, they are both equally, but it's more the drug use that some of these parents have that's causing a lot more damage. (Local authority childcare practitioner, I)
Practitioners, in particular, rationalised their lack of opportunity for further action by describing how they had to balance other more immediate child protection issues, namely the risks to children associated with parental drug and alcohol misuse and violence in the home. There was a clear distinction of the need to prioritise immediate risk against the perceived longer term risk to children from SHSE. These debates were contextualised within the challenges of delivering services to families that participants described as having 'chaotic' lifestyles. Indeed, there was a shared understanding that families with chaotic and difficult lives presented complex challenges for professionals working to protect children from harm.

Children (we see) start off at a disadvantaged position. They are physically compromised by parent lifestyle choices. Respiratory compromised, compromised life opportunities. It's part of a package of neglect and disadvantage. (NHS, Primary Care, FG)

Practitioners also suggested that such complexity could influence both their priorities for action and how parents might view the issue. Thus, just as professionals might not prioritise the issue because of other pressing, immediate concerns about a family, so parents might feel that smoking (and their children's SHSE) was not their key priority or biggest problem in their lives.

We are working with families who are very much at the high tariff end of child protection, and they will be dealing with a whole range of issues from poverty to domestic abuse, mental health, drug and alcohol misuse, and I think just, the day to day struggle for some of our families means that smoking is a release, it's a mechanism that they use to, you know, relax and support themselves. (Local authority childcare practitioner, I)

\section{Continuing ethical arguments}

The ethical issues concerning children's SHSE tended to be avoided or sidestepped by policy advisors and practitioners. In previous work, we explored the ethical challenges that practitioners articulated in terms of balancing protecting children from harm, protecting the relationship between client and practitioner and the rights of the adult to smoke within the private space of the home. ${ }^{20}$ We further developed this argument to consider the sociocultural and environmental challenges that many parents face in creating smoke-free homes and advocated a non-victim blaming approach in smoke-free home interventions. ${ }^{19}$ Many of these previous ethical arguments persisted in the data presented here, with practitioners and policy advisors still grappling with children's SHSE as a child protection issue.

You will have the human rights lobby all over you, saying it's, it's my human right if I want to smoke in my house, I should be allowed to, and I think that that is where you have a real, real dilemma, because from what you said earlier about child protection, yeah, it's a child protection issue, but the lobbyists will say it's a human rights issue, and I don't know how you ever, ever come to a compromise from that. (NHS, Senior Nurse Manager, FG)

Some stated that a children's rights perspective might not be helpful. It was suggested that a purist 'rights' agenda might antagonise parents trying to do their best in difficult circumstances, was not empowering and would therefore be unproductive.

I would not probably go down the route of child protection, but if you were going to be very purist about it, then we could probably say it could be, because it's not very strength based, and it's not very empowering, and there are probably ways that, and, whilst it does put the child at risk, it doesn't put them at immediate risk. (NHS, Senior nurse manager, FG) 
Instead, a more nuanced view was expressed about the need to shift the boundaries of acceptable behaviour, and that this should be an incremental process that required broader cultural changes, through shaping public opinion, which would then influence individual parental behaviour.

\begin{abstract}
Children have a right to good health, and there's all sorts of articles that I can quote at you, the key one about a right to survival and development, so yes, anything that impinges on that, impinges on their right, impinges and diminishes enjoyment of that right, so you can make a very purist argument about all of that, I think what you are really trying to get at is, so how do you change behaviour to make sure that children enjoy that right to the maximum, and that for me is a slightly different question, because that for me is about culture change, about the value that we place on children and young people, and that's where you need to shift it. (Senior Policy Maker, I)
\end{abstract}

\section{DISCUSSION}

The main limitation of this article is the small size of the sample of policy advisors and health and childcare professionals, whose views may therefore not represent the wider policy and practice community in Scotland. However, this purposive sample encompassed a range of key stakeholders in Scotland and included perspectives from different regions. The conclusions drawn should therefore be considered within these limitations.

This article develops understanding of the processes involved in the further development of the social denormalisation of smoking in a country with successful smoke-free public places policies. It is therefore of particular relevance to countries at a similar stage of tobacco control. Both policy advisors and practitioners applied a similar ethical analysis to the furtherance of smoke-free homes. Their accounts provide insights into how they balance their acceptance of an ethical imperative to protect children from SHSE against competing priorities to protect children, as well as the tensions involved in extending the surveillance of smokers from public places to the home by government, when this might be considered unacceptable and disempowering of smokers.

This study demonstrates that Scottish policy advisors and practitioners unequivocally acknowledged the risk of SHSE to children. While accepting this is an important public health concern, they presented a dominant discourse of risk of SHSE to children through a lens of political expediency and the limited rights of the state to intervene in the home. This prevailing discourse of political caution in the further development of smoke-free places is similar to the New Zealand policy makers' discourses of reluctance and resistance when public health intervention is sited in the home. ${ }^{8}$ However, there was support for an incremental policy approach, not requiring legislation, which would aim to shift the prevailing culture further towards normalising the unacceptability of exposing children to SHS, as well as a view that a focus on children's rights would not be productive.

Health professionals, as in our previous study, ${ }^{20}$ continued to find it problematic to prioritise children's protection from SHSE as an issue for their clients. It has remained a contentious issue when viewed within the complexity of intervening with families who are perceived to have chaotic and complex lifestyles. The perception that smoking is a stigmatised health behaviour still appeared to be embedded in the culture of health professionals' practice. ${ }^{11} 20$ The rationale for protecting children was centred upon immediate child safety concerns arising from parental alcohol and drug use or domestic violence. SHSE was viewed as a longer term risk to children and therefore not subject to increased prioritisation, particularly given financial and resource constraints in an economic recession. This rationalisation of risk, as immediate or longer term, in turn shaped their professional practice. This was further problematised when the intervention site was considered to be a private space. Nonetheless, professionals valued their knowledge of people's everyday lives and their understanding of the relationship between smoking and social context. Arguably, such professional knowledge is an under-represented element within tobacco control literature. ${ }^{11} \mathrm{~A}$ redefining of the value of health professionals' tacit and experiential knowledge of their clients' social context may further understanding of the cultures of practice, which are an essential component of denormalising smoking. ${ }^{25}$

The rationalisation of the relative risk of SHSE to children in the home by policy advisors and practitioners leads to a reconsideration of the logic of the prevailing ethical position in policy and practice. An analysis of the ethics of US policy to protect children from SHSE commented that children are less protected in their own homes than in public spaces and questions the logic of this ethical position. ${ }^{26}$ It is evident from our study that children remain, to some extent, unprotected from SHSE in the home by both the policy and practice arenas. The statements about the ethical justification for policy action were made in the context of a country that has successful smoke-free public places legislation and significant social denormalisation of smoking. However, it appears that policy advisors were wary of introducing measures that protect children's health as a right and children's welfare was somewhat avoided. The perceived more immediate risks to children-parental drug and alcohol use, physical abuse and the subsequent demands on health professionals-predicated against the prioritisation of protecting children from SHSE despite evidence of harm. Furthermore, the problematisation of intervening in the private spaces leads to the implicit privileging of parental autonomy to smoke in their own homes over children's rights to health. ${ }^{27}$ The Scottish position resonates with a similar ethical analysis of US SHSE policy and practice, which was found to be inconsistent across states and generally somewhat flawed. ${ }^{26}$ The US authors also concluded that protecting the autonomy of parents to smoke was privileged over the rights of the child to health, even though parental autonomy would only be temporarily curtailed by not smoking in front of children in the home. They argued that the beneficence accorded to children from their protection from SHSE far outweighs arguments about loss of parental autonomy. If the social norms towards the unacceptability of tobacco use and SHSE are to shift further and the momentum of the success of smoke-free public places is to be maintained, it is incumbent on public health policy and practice to counter prevailing arguments about the importance of parental rights to smoke in the home, albeit retaining a non-stigmatised and empowering public health approach.

Interestingly, participants did not reflect on how the car is already a regulated environment in the UK, with laws on using seat belts and mobile phones. These legislative precedents are not currently considered in relation to SHSE in cars. There is no intention in the new Scottish Tobacco Control Strategy ${ }^{28}$ (published after the interviews were conducted) to introduce legislation to prevent SHSE in cars. However, there are new objectives to move the agenda forward through raising public awareness through social marketing campaigns. We also suggest that media advocacy could be used to further the debates outlined here about the lack of children's autonomy to have protection in their homes and cars from SHSE, emphasising that most parents wish to protect their children from harm. ${ }^{14}$ Other countries in the UK are also debating the benefits of introducing legislation to protect children from SHSE in cars and a private member's bill has recently been introduced on this in Scotland. Given the current emphasis on alcohol as a public health priority, it may be possible to explore the synergies between tobacco 
and alcohol policy and public health interventions; however, we would argue that it is important to sustain tobacco control alongside other public health priorities.

\section{CONCLUSION}

This article has illustrated that in a country, such as Scotland, with advanced tobacco control policies there continue to be challenges in progressing policy and practice on SHSE in more problematic and contentious arenas such as the private space of the home. Children's SHSE in the home was unequivocally accepted as a health issue, but was not currently perceived to be a top public health priority in Scotland by policy advisors and health and childcare practitioners. However, there was a consistent narrative accepting the harm of SHSE for children and the need to shift the culture further towards the unacceptability of children's SHSE, particularly within the home. There were several barriers to furthering SHS policy and practice, not least financial cuts that had impacted on organisational priorities for action and the competing needs of addressing parental alcohol and drugs, domestic violence and child abuse, which were seen to be more pressing and immediate child protection concerns.

\section{What is already known on this topic}

- Exposure to secondhand smoke (SHS) is an important cause of morbidity in children, who are most often exposed in their homes, particularly in disadvantaged homes.

- Most parents want to protect their children from harm and many homes have some form of smoking restriction, but these restrictions are often flexible in their implementation.

- Policy advisors and practitioners are challenged by developing public health interventions in the private space of the home.

- The implementation of smoke-free public places in Scotland did not increase children's SHS exposure in the home.

\section{What this article adds}

- Policy advisors and practitioners accepted the harms caused by children's exposure to secondhand smoke (SHS).

- They supported an incremental policy approach, not requiring legislation, which would aim to increase the unacceptability of exposing children to SHS.

- Taking action to protect children from exposure to SHS in the private space of the home was problematic for both policy and practice.

- Protecting children from SHS was linked to competing child protection concerns. Immediate child protection concerns were prioritised over SHS exposure of children in the practice arena.

- Ethical considerations remain unresolved in the policy and practice arenas. The right to health for children does not prevail over the autonomy of parents to smoke in the private space of their homes.

\footnotetext{
Author affiliations

${ }^{1}$ School of Health in Social Science, University of Edinburgh, Edinburgh, UK ${ }^{2}$ UK Centre for Tobacco Control Studies, Centre for Population Health Science, University of Edinburgh, Edinburgh, UK
}

${ }^{3}$ ASH Scotland, Edinburgh, UK

${ }^{4}$ Department of Environmental and Occupational Medicine, University of Aberdeen, Aberdeen, UK

${ }^{5}$ Department of Child Health, University of Aberdeen, Aberdeen, UK

${ }^{6}$ Centre for Population Health Science, University of Edinburgh, Edinburgh, UK

Acknowledgements We are grateful to all the participants who took part in this study.

Contributors SS, ST, AA and DR designed the study. The project was managed by $A S$ and RO. CM carried out the field work. DR wrote the first draft of this article. AA is guarantor for the study. $D R, C M, A A, A S$ and $R O$ were involved in the data analysis and $D R, C M, A A, A S, R O, S S$ and $S T$ contributed to this article.

Funding This work was supported by the Big Lottery Fund, grant number RGT/1/ 010332728

\section{Competing interests None.}

Ethics approval The study complied with the code of practice on ethical standards for non-commercial research with the North of Scotland Research Ethics Committees and NHS Grampian Research and Development Office.

Provenance and peer review Not commissioned; externally peer reviewed.

Data sharing statement Participants did not give consent for data sharing and the risk of sharing the data is higher than the potential benefits.

\section{REFERENCES}

1 IARC. Monographs on the evaluation of carcinogenic risks to humans. Evaluating the effectiveness of smoke-free policies. Vol 13. Lyon, France: International Agency for Research on Cancer 2009;136-62.

2 Akhtar P, Currie D, Currie C, et al. Changes in child exposure to environmental tobacco smoke (CHETS) study after implementation of smoke-free legislation in Scotland: national cross sectional survey. BMJ 2007;335:545-9.

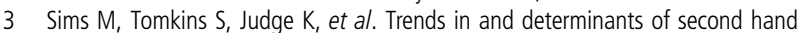
smoke exposure indexed by cotinine in children in England from 1996-2006. Addict 2010;105:543-53.

4 Jossens L, Raw M. The Tobacco Control Scale 2010 in Europe. Brussels: Association of European Cancer Leagues, 2010

5 Donnelly P, Whittle P. After the smoke has cleared-Reflections on Scotland's tobacco control legislation. Public Health 2008;122:762-6.

6 Haw S, Mackie L. Fresh air. In: Barlow J. ed Substance misuse, policy and practice implementation. Research highlights in social work. London: Jessica Kingsley, 2009:53-61.

7 Bauld L. The Impact of Smoke-free Legislation in England: Evidence Review. University of Bath for Department of Health 201:1-20 https://www.gov.uk/ government/uploads/system/uploads/attachment_data/file/216319/dh_124959.pdf (accessed 29 Jul 2013)

8 Rouch G, Thomson G, Wilson N, et al. Public, private and personal: qualitative research on policymakers' opinions on smokefree interventions to protect children in 'private' spaces. BMC Public Health 2010;10:797.

9 U.S. Department of Health and Human Services. The Health Consequences of Involuntary Exposure to Tobacco Smoke: A Report of the Surgeon General. Rockville, MD: U.S. Department of Health and Human Services, Public Health Service, Centers for Disease Control, Center for Health Promotion and Education, Office on Smoking and Health. DHHS Publication No. (CDC), 1986.

10 U.S. Department of Health and Human Services. The Health Consequences of Involuntary Exposure to Tobacco Smoke: A Report of the Surgeon GeneralExecutive Summary. U.S. Department of Health and Human Services, Centers for Disease Control and Prevention, Coordinating Center for Health Promotion, National Center for Chronic Disease Prevention and Health Promotion, Office on Smoking and Health, 2006.

11 Ritchie D. Creating smoke-free environments: public and private spaces. Unpublished PhD by publications, University of Edinburgh, 2012.

12 Oberg M, Jaakkola M, Woodward A, et al. Worldwide burden of disease from exposure to second-hand smoke: a retrospective analysis of data from 192 countries. Lancet 2011:377:139-46.

13 Akhtar P, Haw SJ, Currie D, et al. Smoking restrictions in the home and second-hand smoke exposure among primary school children before and after introduction of the Scottish smoke-free legislation. Tob Control 2009;18:409-15.

14 Philips R, Amos A, Ritchie D, et al. Smoking in the home after the smoke-free legislation in Scotland: qualitative study. BMJ 2007;335:553-7.

15 Ayres JG, Semple S, MacCalman L, et al. Bar workers' health and environmental tobacco smoke exposure (BEHTSE): symptomatic improvement in bar staff following smoke-free legislation in Scotland. Occup Environ Med 2009;66:339-46.

16 Pell JP, Haw S, Cobbe S, et al. Smoke-free legislation and hospitalizations for acute coronary syndrome. N Engl J Med 2008;359:482-91.

17 Akhtar $\mathrm{P}$, Haw S, Levin K, et al. Socio-economic differences in second-hand smoke exposure among children in Scotland after introduction of the smoke-free legislation. J Epidemiol Community Health 2010;64:341-8. 
18 Jones LL, Atkinson O, Longman J, et al. The motivators and barriers to a smoke-free home among disadvantaged caregivers: identifying the positive levers for change. Nicotine Tob Res 2011;13:279-486.

19 Robinson J, Ritchie D, Amos A, et al. "Waiting until They Got Home": gender, smoking and tobacco exposure. Soc Sci Med 2010;71:884-90.

20 Ritchie D, Amos A, Phillips R, et al. Action to achieve smoke-free homes-an exploration of experts' views. BMC Public Health 2009;9:112-20.

21 Wilson I, Semple S, Mills LM, et al. REFRESH—reducing families' exposure to secondhand smoke in the home: a feasibility study. Tob Control 2013;22:5 e8.

22 Wilson I, Ritchie $D$, Amos A, et al. 'I'm not doing this for me': mothers' accounts of creating smoke-free homes. Health Educ Res 2013;28:165-78.
23 Mills LM, Semple SE, Wilson IS, et al. Factors influencing exposure to secondhand smoke in pre-school children living with smoking mothers. Nicotine Tob Res 2012;14:1435-44.

24 Ritchie J, Lewis J. eds. Qualitative Research Practice A guide for social science students and researchers. London: Sage 2003:24-46.

25 Fisher $\mathrm{P}$, Owen J. Empowering interventions in health and social care: recognition through 'ecologies of practice'. Soc Sci Med 2008;67:2063-71.

26 Jarvie JA, Malone RE. Children's secondhand smoke exposure in private homes and cars; an ethical analysis. Am J Pub Health 2008;98:2140-5.

27 United Nations Convention on the Rights of the Child, New York, United Nations, 1989.

28 Scottish Government Tobacco Control Strategy- Creating a Tobacco-Free Generation. Edinburgh, Scottish Government. 2013. http://www.scotland.gov.uk/Publications/ 2013/03/3766 (accessed 29 Jul 2013). 